This item was submitted to Loughborough's Research Repository by the author.

Items in Figshare are protected by copyright, with all rights reserved, unless otherwise indicated.

\title{
Integration approach to virtual-driven discrete event simulation for manufacturing systems
}

PLEASE CITE THE PUBLISHED VERSION

https://doi.org/10.1080/0951192X.2014.924159

\section{PUBLISHER}

(C) Taylor \& Francis

\section{VERSION}

AM (Accepted Manuscript)

\section{PUBLISHER STATEMENT}

This work is made available according to the conditions of the Creative Commons Attribution-NonCommercialNoDerivatives 4.0 International (CC BY-NC-ND 4.0) licence. Full details of this licence are available at: https://creativecommons.org/licenses/by-nc-nd/4.0/

\section{LICENCE}

CC BY-NC-ND 4.0

\section{REPOSITORY RECORD}

Ghani, Usman, Radmehr P. Monfared, and Robert Harrison. 2019. "Integration Approach to Virtual-driven Discrete Event Simulation for Manufacturing Systems”. figshare. https://hdl.handle.net/2134/26771. 


\title{
Integrating Approach to Virtual Driven Generic Discrete Event Simulation for Manufacturing Systems
}

\author{
Usman Ghani, Radmehr Monfared, Robert Harrison \\ Wolfson School Loughborough University UK
}

\begin{abstract}
Manufacturing systems designers and planners ever tries to adopt the modern technologies and techniques to design and validate different production processes. These modern techniques help to facilitate the better, faster, cheaper and reliable products in market to meet the customer demands. It is very useful if the manufacturing system/assembly line performance to be verified in the early stage of resource planning. Strong collaboration between the machine user and its builder can make it possible. Virtual environments are the new emerging approaches which not only provide the collaboration but also help in the resources design and verification based on the product and processes. The machine builder and its user can reconfigure the machine setups from its components in virtual model in an agile and concurrent mode. To examine the impacts of reconfigured machine components activities at low level on the high level business productivity there must be a link between the virtual environment and discrete event analysis. Therefore to integrate the virtual engineering tools data with the discrete event simulation this paper proposes an approach of virtual driven generic discrete event simulation modelling (named as VDsim as a packaged tool) to facilitate solid decisions prior to production with the help of real times generated by virtual machines. The success of this discrete event simulation analysis depends on the quality of input data its compatibility and integration process. The data link needs to have a seamless interfacing between these independent applications in a generic form to increase models reusability. This integration package not only brings these two applications together but also facilitates the production planners and schedulers to examine the most possible optimum status for the resources on the production line in-term of the cost, energy consumptions and impacts of the machines reliabilities on throughputs.
\end{abstract}

Key Words:

\section{Introduction}

Modern manufacturers need to have the ability to configure, validate and optimise their manufacturing processes according to the market dynamics [1].DES is method to observe the impacts of machines processes re-configurability on layouts, processes and resources allocations for new or existing production setups [2]. Different DES scenarios building applications such as Arena [3], Witness [3], and Simul8 [4] are commercially available. Discrete event simulation modelling provides a quick and low cost method to asses the risks associated with the changes in resources setups at the very early stage of production line development[5]. DES models applicability could be enhanced if it is developed in a generic form. This is possible if consistent processes related real time data integration specification 
is available throughout the domain being simulated. To get the advantage of early validation and design of resources for what-if conditions a real time of machine operations must be generated in an application which could eliminate the physical build of the machine prior to be commissioned and tested.

This is possible if the production processes are design and visualise in a virtual environment which provides a view on machines used in production line. Also VE is a common platform for both the machine user and its builder to verify all of the processes and its related data virtually upto the components level. Different tools such as Delmia [6], Tecnomatix [7] and Core Control Editor (CCE) are the example of virtual engineering tools. These tools are either generic or customised for specific application domain.

The virtual Engineering tools, in particular CCE which has been analysed in this research, can design and visualise systems based on a modular component structure and can provide the opportunity to represent a complete machine or production facility after assembling components in a virtual environment. The independent motion of each component in machine represent a process as per actual machine in line[8]. Therefore there is a potential to utilise the dynamic data generated by the components of the visualised machine for the discrete event simulation. It could bridge the low level reconfigurability effects because of the different possible arrangements of the machine components at the higher level decision. In addition, the virtual tools could potentially facilitate discrete event simulation tools to address many important production issues such as machine breakdowns, repairs and energy consumptions at component level during the discrete event simulation model analysis.

The focus of this paper is to report the research carried out to enhance the capability for early validation and system design of different production scenarios for end user through generic and reusable discrete event simulation model, driven by data generated in a virtual engineering environment. To have a generic/re-usable discrete event model, it is important to have the process, resource and data flexibility during model development, which is possible if a standard data structure e.g. XML[9] is used to model the virtual engineering tools data for the production line.

Proper data structuring could be achieved for DES models with the help of unified systems decomposition across the production life cycle and sufficient information manipulation. Different approaches and tools have been developed and used by various research groups to achieve such integrations. The Unified Modelling Language (UML)[10] is one of the commonly used approach to formalise capturing, modelling and visualising the process decomposition and associated information systems over the production lifecycle. This modelling approach could enable visualising the interaction between the system components and data flow pattern to run the discrete event simulation model. Understanding the data acquisition and its interface to discrete event simulation allow the user to generate a generic model applied to different application tools within a similar domain.

To develop an effective working model for a discrete event simulation driven by data generated in a virtual engineering environment, system integration, hierarchical system design and their implementation issues should be investigated. Both virtual engineering and simulation approaches are used widely in various industrial sectors, in particular automotive industries. The approach adopted in this paper is especially focused in automotive sector.

\section{State of the Art Research}

With the advancement in technology and tough market competition, business managers need to have real time business and operational information to support business decisions. 
The virtual engineering tools (VE) such as CCE can facilitate accessing such information through early visualisation and virtual design, whereas discrete event simulation (DES) models provide means of predicting the system operational behaviour typically through what-if scenarios.

An integration process must be developed between the VE and DES to utilise the real time virtual data to make a close decision process between product producer and machine builder to optimise the production resources. Thus the discrete event simulation model driven by the virtually generated data is a significant advancement in this regards. Data flexibility about the DES models could increase the effectiveness of a model, which could help to make easy what-if scenarios for a decision process [12].

Pidds [13] suggested working on data driven simulation in 1992. One of the other possible option is to develop simulation model from the process based charts to control the model development complexity and time requirement, such as the method proposed by Lee [14] in 2000. Many other efforts like NIST research group made for data collection from shop floor in a generic and structured form[15]. Bengtsson et al paid an effort for automatic simulation model running from captured data focusing to increase the reusability of the model [16]. To have the reusability of data a common setup of processes and data is required to be used for the resources employed on the production line. Virtual environments are the tools which could be used for the standard data set generation in form of XML along with the visual processes verification and validation[17]. Therefore VE not only help to visualise and validate the product process design but also develop the geometric simulation [18]. Many Virtual tools are available to be used in the production processes planning and design e.g. Delmia, V-Sim, UGX technomatix, Core Control Editor (CCE at Loughborough) etc[19]. These virtual scenarios tools need to have a well updated data. The timing information generated on the bases of this data can be used in different applications analysis typically in the real time discrete event simulation modelling to observe the behaviour of production line. In large scale production and agile reconfigurable manufacturing virtual tools are very supportive to validate the operations at machine level for different configurations I[20].

To enhance the utilisation of virtual tool in the production line analysis prior to production it is suggested to have a proper generic valid for a range of softwares. Interface with the discrete event simulation software.

To have a generic integration approach suitable for different virtual environments and DES applications this research is mainly focused on the tool design (VDSim) linking the processes data and information of VE with the DES models. The National Institute of Standards and Technology worked in the Core Manufacturing Simulation Modelling Information collection for shop floor using the UML model mapping. And these standards are accepted at SISO in 2010[21], which could provide a background for the data manipulation required in the discrete event simulation modelling driven by virtual data.

[22]. Close loop integration based on the flow of right information required for analysis, design and production processes analysis. [22]. Systematic categorisation of right information is backbone for bringing generity in an approach. The UML modelling methodology could help to capture static information as well as behavioural data of the machine components[22].

Presently discrete event simulation models are driven by the shop floor data and the generated results help only in predicted decisions. But there is a potential to utilise the virtual tool behavioural information for the line simulation modelling, based on the real time data. Integrating virtual tools information and data with discrete event simulation will present a 
complete package supportive for the independent user with less complexity and low cost along with the software's and data flexibility.

\section{Frame Work of System Design and Life Cycle Analysis}

This research focuses on automotive industry as a use case domain to experiment the proposed concept of integrating VE environment to the simulation models. Figure illustrates typical interaction of business collaborators involved to produce an automobile engine as a product. The collaborators identified in this business sector, are end users, machine (or facility) builders, and suppliers or vendors. The end user is the engine manufacturer (market product), the machine builder designs and produces production and automation facilities, and the suppliers are (in this use case) the vendors who provide materials, components and software applications required to build a machine.

Figure 1 shows the conventional level of interaction between the different departments within the end user organisation, machine builder and suppliers.

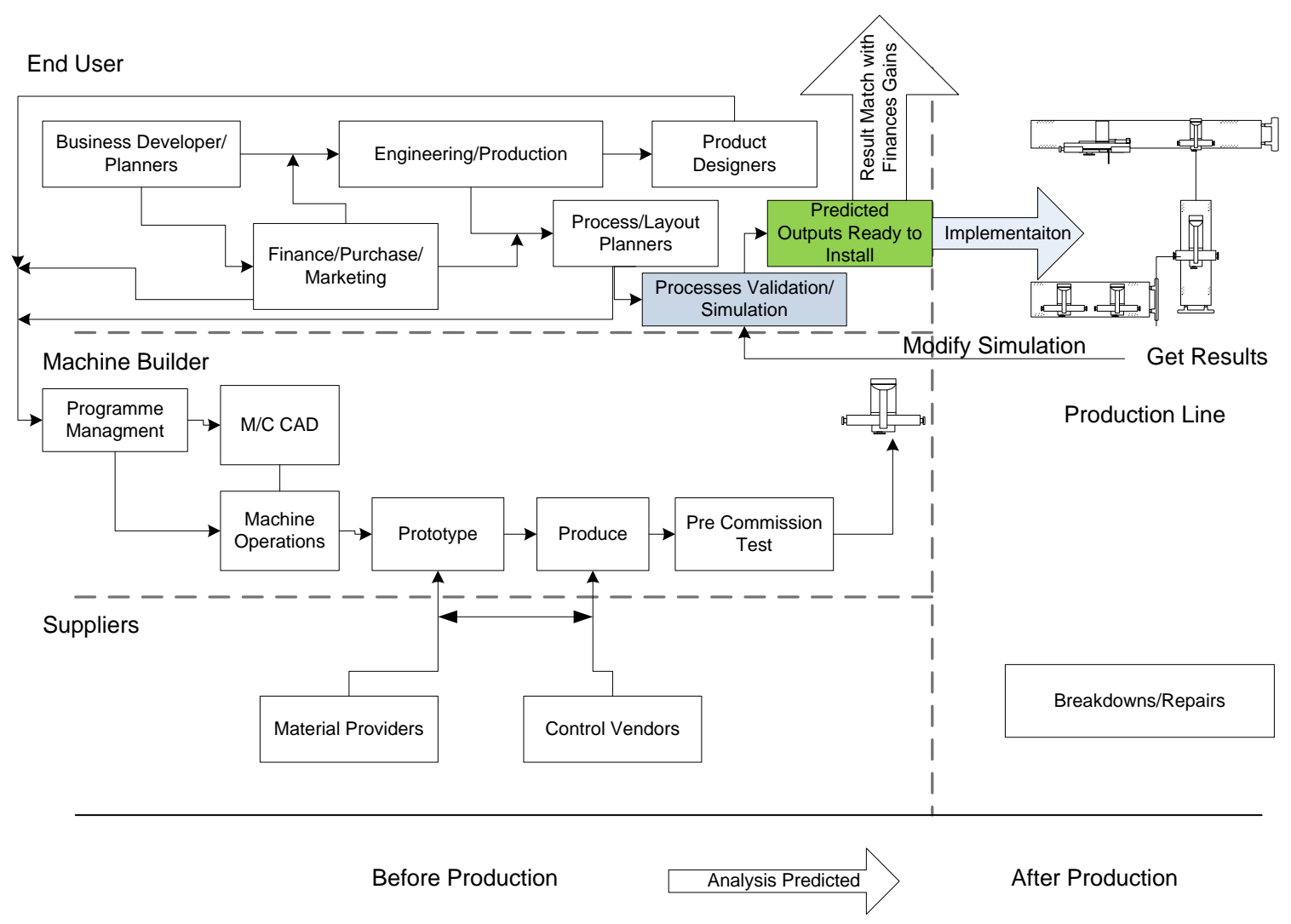

Figure 1: Conventional System Design approach

On end user side the business developers set the business targets either to update the existing production setups with new technologies or to install the new production units in case of business extension. The business developers take their finance, product design and production departments on board to set the suitable business targets. On the basis of the preliminary feasibility studies various department initiate the design of the production systems. Some of important tasks include defining the process layout and process sequence 
for different operations in an optimised manner to avoid any non-value adding activity in the production. Typically processes verification and validation are supported with the discrete event simulation models.

At the same time the end user finance/purchase department interacts with the machine builder to initiate the machines build. The machine builders with the collaboration of the end user's product design and process developers start working on the machine design and build. A prototype is typically build in case of new machines building to validate and examine the cycle times. At this stage, the supplier of material and control systems (required in machine automation) interact with the machine builder as the third tier collaborators.

The build of the production facilities is started once the prototype is accepted by the end user's design engineering group. A number of pre and post commissioning activities are included in the business process to increase the chance of early detection of the design or manufacturing faults. The pre-commissioning test are typically carried out at the builder site and approved by the end user's process, layout and planning engineering teams. Following installation of production line the processes and line outputs impacts on the business targets are checked again in the discrete event simulation for the second time to identify non value adding activities based on the actual cycle times and activities after the production.

The conventional systems design approaches lack an effective decision support system to enable end users to predict the system behaviours before manufacturing and installation. Typically, historical data (from previous projects) are used to estimate the system behaviours, performance and capabilities. This method is not free from errors and heavily depends on the individual's experiences.

The critical situation is take place when multiple changes occur to the product or the production facilities' design during the build process. These changes may be market driven start in the 2 or 3 years of new engine programme, or simply because of design errors. The initial impacts of implementing changes are usually high costs and causing significant delays in production schedule.

To handle these limitations in the last 10 years automotive industry has moved towards more generic and reusable designs for the manufacturing facilities and extensive use of simultaneous engineering and virtual design environments. As a result a significant improvement has been made by demonstrating and understanding manufacturing and commissioning problems as early as product and production system design stages.

Figure zillustrates part of the more recent application of virtual engineering tools within the automotive industry. Using virtual engineering approach the engineers will have a good understanding from the system capabilities and their behaviours under the business production parameters. In addition, the physical prototyping before the machine build can be significantly reduced (or eliminated in some cases). This could be possible by embedding the process behaviours and geometrical visualisation of machine components into the virtual design.

As shown in the figure, the virtual tools can be used in the physical resource building process as well as in the production planning and validation. This produces a strong collaboration among all of the partners regarding the production line outputs and processes verification for the end user. It is equally effective for the machine builders by avoiding development and predicting faults in the design of the resources and cycle times in the 
conventional methods. This could save significant time and cost by minimising the machines prototyping processes.

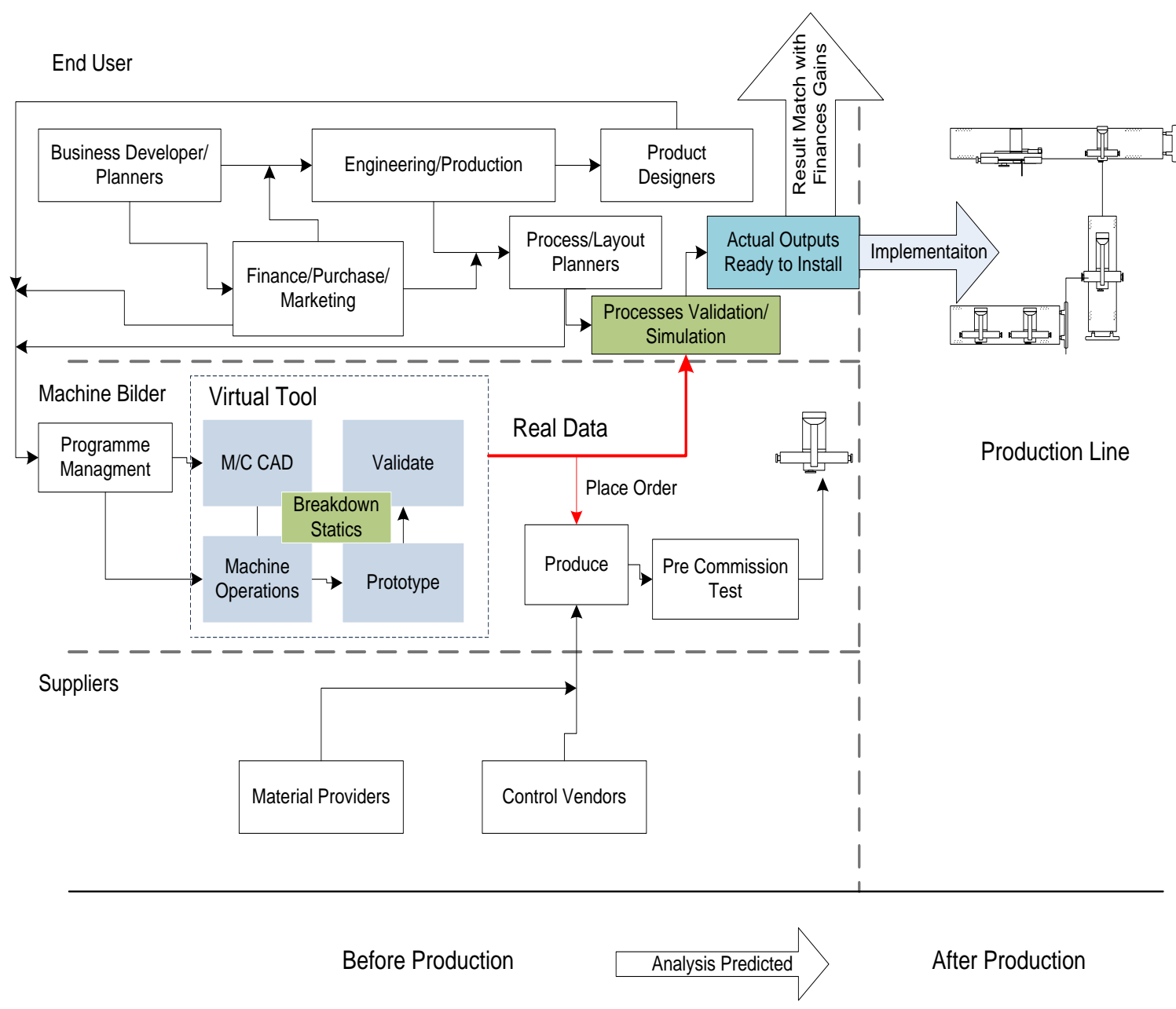

Figure 2: Application of virtual engineering and proposed integration to the simulation models

Despite a limited use of virtual engineering within industries, the data developed by the VE tools are not currently exploited efficiently throughout the business life cycles.

In addition to the visualisation and early design error detections, some of the more advanced VE tools such as CCE has a potential to produce data required for an accurate discrete event simulation. Such integration (see figure 2, proposed integration) will enable early access to the key system capabilities, and performance data required for optimising production systems through discrete simulation methods (link is shown by the dark and bold line).

As the virtual tools support the replication of actual physical systems and resources, it is proposed that the VE generated data to be used for development of more accurate simulation models that facilitate decision making and estimating the system performances as early as the design stage.

This emerging approach could help to verify and match the low level machine components activities at shop floor with the business targets set by the business management. The end users can potentially get the advantage of first time right results from discrete event 
simulation which supports management to devise more accurate plan of actions about the production performances and the financial gains.

The production planners and process engineers will be able to analyse the manufacturing and assembly lines for financial analysis, bill of material, human resource utilisation, machine associated energy consumption and machine performances.

In addition, some of the production line variables such as machine breakdowns and preventive maintenance schedule can be incorporated to the VE data structure for the DES use.

\section{Proposed Concept}

The proposed concept of integration between the VE and DES to run the discrete event models described in this article is illustrated in Figure 3.

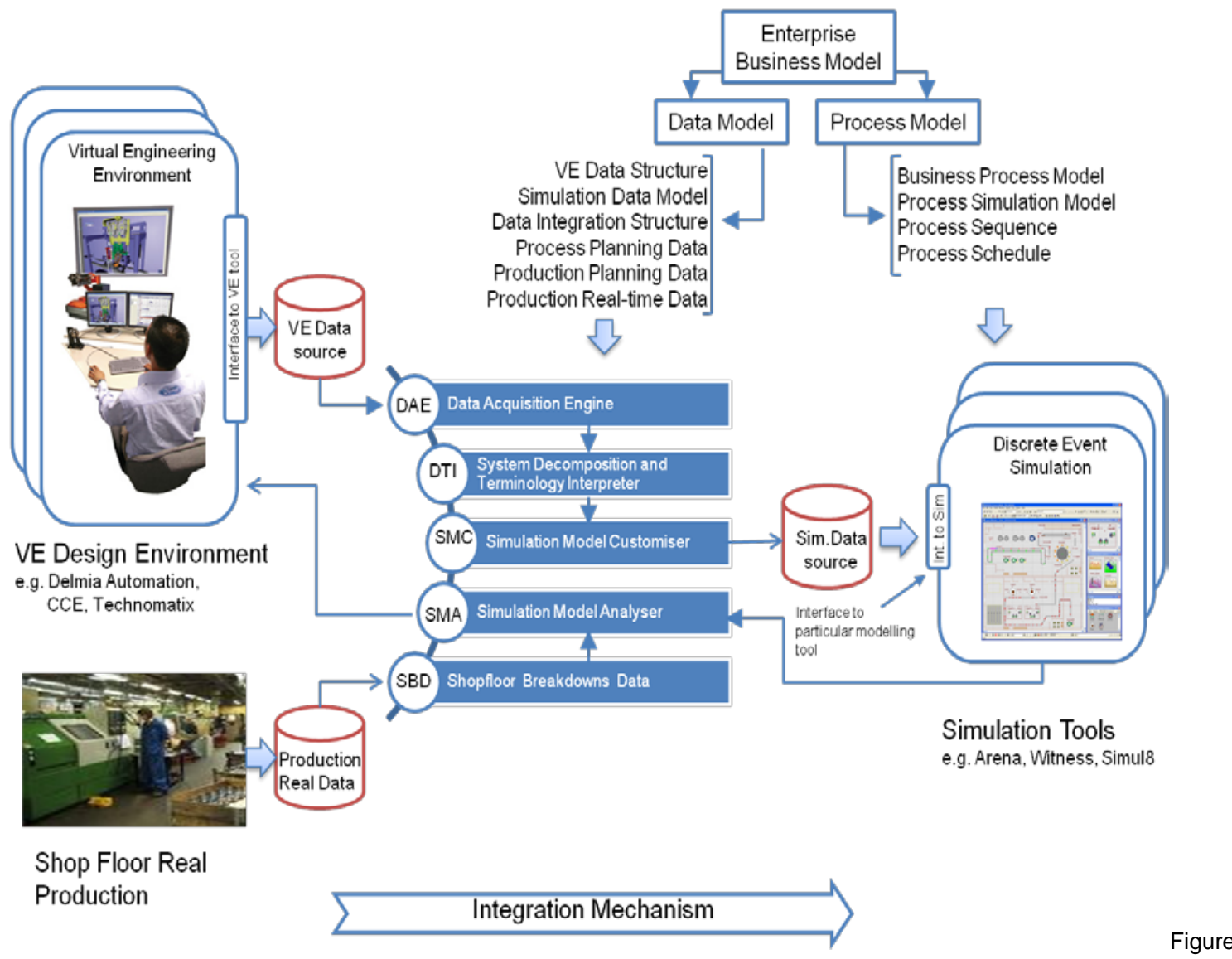

3: Concept of System Design and Integration

Two sources of data are required within the proposed framework shown in Figure 3, the virtual engineering environment generated data and the operational data from machine at the shop floor. The VE data source is captured, filtered and partially used to drive the simulation model. However, the actual productions parameters are observed and collected from the shop floor. This data has the information about process planning, production planning, actual cycle times and data structure. 
The proposed concept comprises data model and process models, generic simulation modules, and a number of functional modules embedded into one application tool collectively known as VDSim. The VE data specifies the customisation of the generic simulation models according to the virtual system design. The data structure and the data transactions are organised by the data model, whereas the process sequence, the operational conditions (e.g. interlocks) and the resource requirements are defined by the process model.

The "Data Acquisition Engine" (DAE) is developed to analyse and capture data sets required for the simulation models. The structure, types, and decomposition of the VE data is in nature different from the data required for the simulation models and therefore an algorithm was developed as a functional module called "System Decomposition and Terminology Interpreter" (DTI) to interpret and translate the VE data. The simulation model is developed with a generic structure, customisable via simulation parameters, managed by "Simulation Model Customiser" (SMC). The result of the simulation model may identify necessary changes to the VE library of reusable modules. Such changes are highlighted by the "Simulation Model Analyser" (SMA). The SMA basically compares the simulation results with the default parameter values recorded in the VE library and make recommendations for potential amendments. The production data is captured and filtered by the "Shopfloor Breakdowns Data" (SBD) module, which currently focused on the breakdown data only.

The proposed integration functionalities (VDSim) concept is described in further details in the following section.

\section{System Integration Design (VDSim) Functionalities}

Main issues to be considered about to use the virtual tools data in the DES models, are data compatibility and data integration. This section addresses different aspects of integration aspects between virtual engineering and simulation modelling to analyse and validate the production model running based on data generated by a virtual design tool.

\subsection{Data Modelling}

It is most important to have a systematic approach to model a structured data inputs particularly for virtual tool driven discrete event simulation. The NIST group in USA contributed efforts for shop floor data categorisation which is still towards the maturity in this regard $[15,23]$. Such a proper standard information and data infrastructure enhances the capability of the DES models driven by VE environments. Also it helps to develop the standard interfaces for automatic models running from the VE data.

For data associated information modelling this research work adopted uses unified modelling language methods. The UML manipulates the informations as well as decompose the systems properly to synchronise the right data. These standard steps about the informations and data helps the system designers and integrators to apply this research work for a variety of virtual environments. 
Initially, the shopfloor data model is constructed based on the processes, resources, parts and the resource organisation, as shown in Figure 4. For example if a machine station analysis is required in production line therefore the information regarding its type, the setups times, repair loops, energy consumption, schedules etc. have to be captured. The standard way to access these static information is the UML class model along with the associated attributes used for each component of the machine, which typically perform a production process.

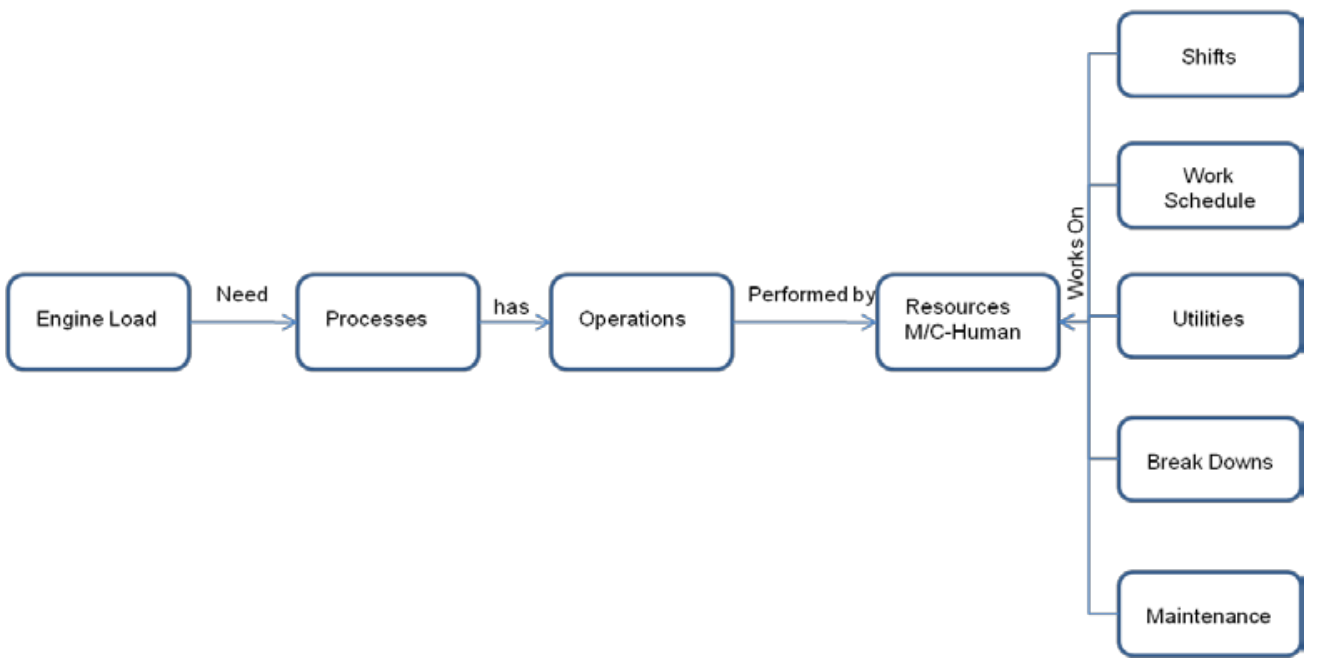

Figure 4 Example of Required Information and Data

Typically Figure 4 represent the desired information required to build the class model in UML prior to provide the inputs at components level of machine in the virtual environment during the machine station design. The figure is an example of the informations of the automotive engine loading station to perform different processes. These processes have some independent operations which use resources of different type's e.g. automatic machine centre/semiautomatic/manual along with a human interaction. These resource has the information about its working scenarios like shifts patterns, utilities consumptions and reliability concerns etc.

The structured information could help to get the right data suitable for the DES model. To access the right data there must be a right match between the two different applications. This research addresses the match integration between the VE and DES at the process level. Therefore a proper decomposition of the two applications is required to understand the process and data interaction. Figure 5 illustrates the level of interaction and information flow pattern used in UML. 

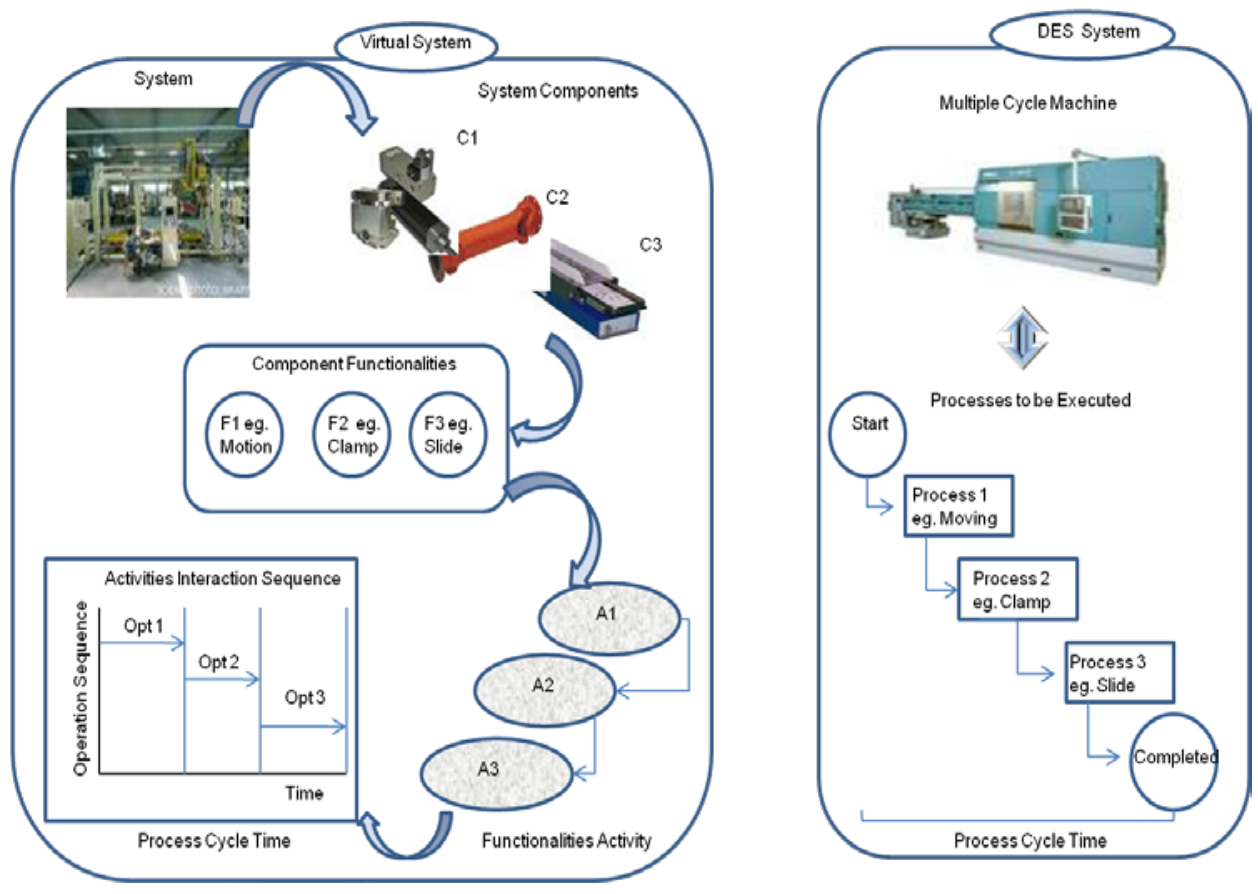

Figure 5 Data Modelling Level and Virtual System Decomposition

This figure has been devised on the resemblance between the UML information decomposition and the VE system components interaction and data generation level. Like the UML use case model figure shows the machine components in the virtual system which exhibits some functions e.g. fastening, moving etc. Like the activity interaction diagram of UML there is a specific activities interaction exist between these functionalities followed by a proper process sequence having the timing information (cycle times etc) of the processes as compared with the sequence chart of UML.

On the other side the DES could capture the process sequence in order to analyse the processes performed by the components of a machine in a discrete manner. At the process sequence level now both the tools are compatible with each other. The VE tool could generate a structured XML data file of these processes in term of the cycle times and other associated data like machine breakdowns etc. which is interpretable with the help of VDSim (DAE, DTI, SMC, SMA and SBD) integration tool in the DES model after following some functions as shown in Figure 3.

\subsection{Data Acquisition/ Compatibility Engine}

The nature and structure of the data used and generated within a virtual modelling environment is different from data required for a data driven simulation system, which need manipulation to streamline.

The VE tools typically produce sets of data for example process times number of resources, breakdowns and shift data related to the system's run-time behaviours. A standard approach to the information modelling is required to structure data before applying various data mining algorithms to extract data required to drive the simulation model as said in earlier section using UML approach. 
After investigating a number of virtual design tools, a standard $\mathrm{xml}$ data format is adopted to exchange the data from one modelling domain to another. The modelling data of the virtual design tool (in form of $\mathrm{xml}$ format) as an output is sorted by Data Acquisition Engine (DAE) developed as part of this research. The DAE module working is shown in Figure 6.

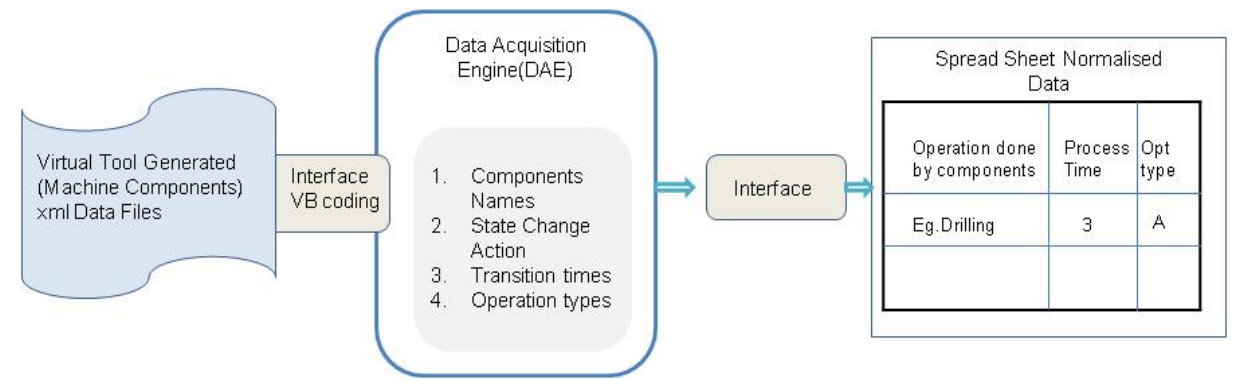

Figure 6 Data Acquisition Engine (DAE) Extract VE Data from XML File

DAE basically extracts the required data and manipulates it according to the required information as an output in a spreadsheet. Typically machine components names, their states transitions, and times taking to move in these states along with the type of operations are acquired from the XML data file.The raw data is normalised in the form of process sequence, times and operation types. The normalised data is then formatted on the process model being developed in the discrete event simulation. While individual data interface should be designed for various VE application tools. As part of this research interface to CCE tool has been developed.

\subsection{Applications Data Terminology Interpretation (DTI)}

The data structures and terminology used in a number of research based and commercial VE tools were studied in detail. It was understood that there is a lack of data compatibility between the virtual engineering tools and discrete event simulation models. Each VE tool has its own proprietary terms, phrases and system data decomposition method, which are typically deployed for specific industrial application domain. However, further study proved that with appropriate interpretation and omitting certain level of excessive details from various tools, a method can be developed to interpret the terminology to a unified format suitable for the discrete event simulation models.

Examining a number of different tools including Delmia Automation, CCE, and Technomatix a large collection of typical components and terminology for modelling objects were captured. On the other hand the data classification and modelling terminology for discrete event simulation were also structured. Table 1 illustrates part of the results from using VE machine station decomposition and terminology interpretation used in this research. 
Table 1 VE tool Terminologies List Comparison with DES

\begin{tabular}{c|c}
\hline Virtual Tools Terminologies & Discrete Event Simulation Terminologies \\
\hline System & Machine \\
\hline Route & Path \\
\hline Component & Cycles/Multiple Cycle \\
\hline Time & Labour \\
\hline Manikin & If-Else Statements \\
\hline Sensors & Breakdown and Repair \\
\hline System Reliability & In-Out Logic \\
\hline Complete Process/Job & Module \\
\hline Module & \\
\hline
\end{tabular}

Table represent the typical terms used in VE and discrete event simulation tools e.g. in CCE and Witness tool. This understanding especially provides the flexibility for the user to integrate mutual terms used in different working applications.

The data terminologies interpreter (DTI) has been developed to perform both the decomposition and the interpretation of terminologies as shown in Figure 7.

This DTI module developed in this study is optimised for CCE virtual engineering tool. The conversion of the CCE tool term to the Witness simulation model is developed as a proof-ofconcept model in this study.

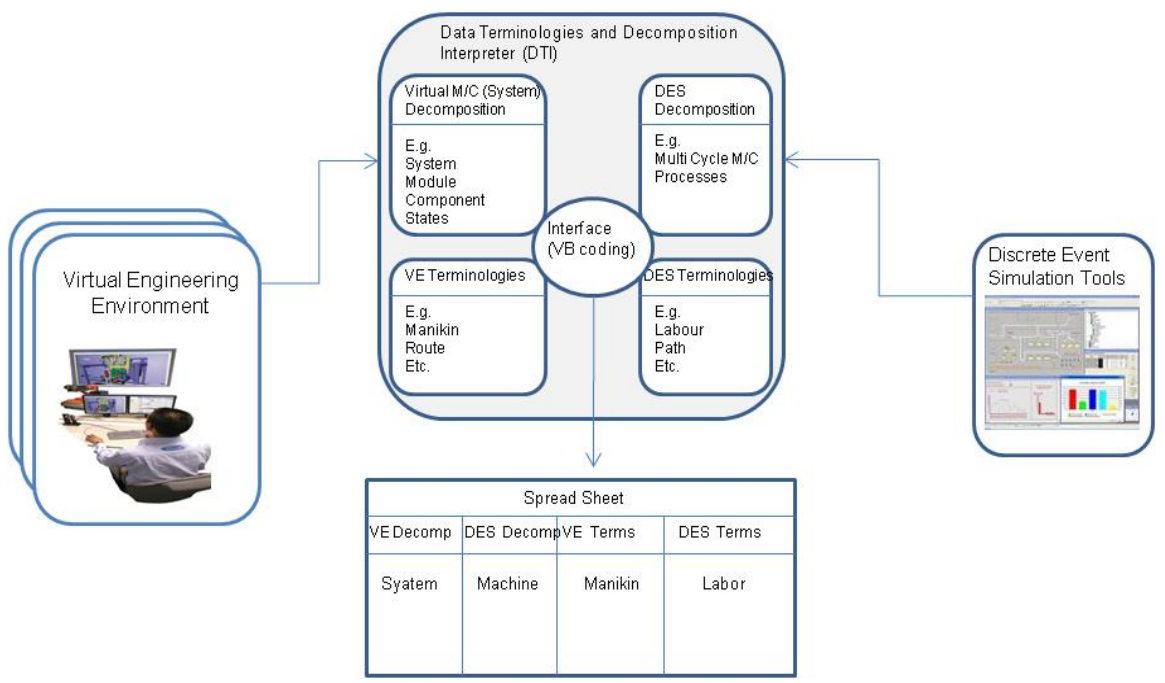

Figure 7 Data Terminologies Interpreter

The data structure, terminology, and the data hierarchy (or data/process decomposition) of the VE and simulation tools are predefined and embedded into the module for specific tools. An internal logic has been coded in the module that receives data from VE models and translates them to data sets suitable for simulation modelling. The conversion interface is 
specific for a VE and a simulation tool and should be updated for various tools. In this study, the VE translated data is stored in a spread sheet to be fed to the simulation model.

\subsection{Simulation Model Customiser (SMC)}

The simulation models are structured in a generic manner with a set of variable parameters as input and outputs. The variable parameters are stored in a spread sheet controlled by the "Simulation Model Customiser" (SMC) module. The SMC provided interface to the end users to customise the simulation model based on various what-if scenarios as shown in Figure 8 . In addition, the SMC module customise the simulation model for specific analyses such as estimating or monitoring (when running in real time) energy consumption at component levels, or calculating system utilisation based on the predicted required resources and the estimated breakdowns.

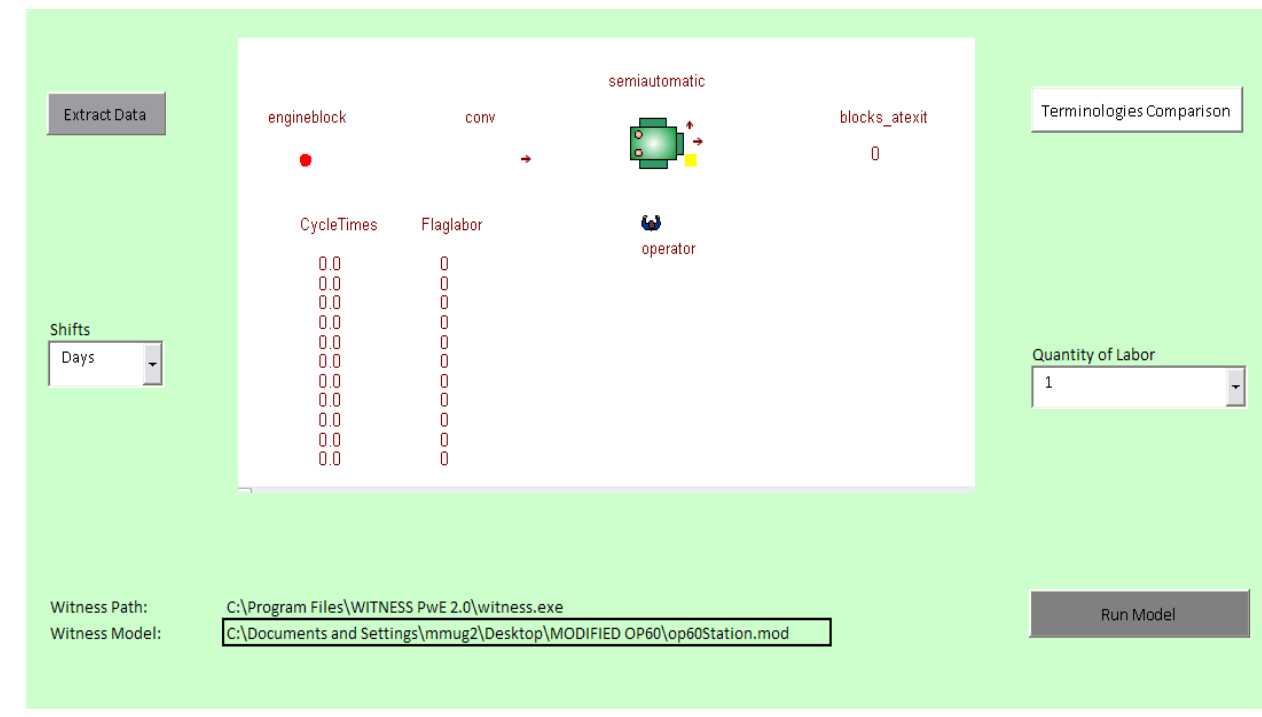

Figure 8 Example of Simulation Model Customisation

\subsection{Simulation Model Analyser (SMA)}

Following customisation of the simulation model the output data vary accordingly. The simulation generated data is collected by the "Simulation Model Analyser" (SMA) to be filtered and formatted for specific uses. This data provide system performance information and system operational details (e.g. time, cost, breakdowns, number of resources, etc.). Furthermore, the SMA module has been optimised to highlight misalignment and potential errors in VE data based on the simulation runs. Example of potential errors may be the overall achieved cycle time when queuing and buffering are taken into consideration (queuing typically is not considered in VE tools).

\subsection{Shop Floor Breakdowns Data (SBD)}

Theoretically, the simulation model developed with the help of VE generated data has the information about the system reliability defined by the machine builder for the components 
used in the virtual tool. Thus the simulation results based on this data represent the actual performance data for the physical resources on production lines. However, this is typically not the case as there are many variables which cause unplanned machine breakdowns, repairs and labour inefficiencies. Some of the parameters involved in SBD are shown in Figure 9.

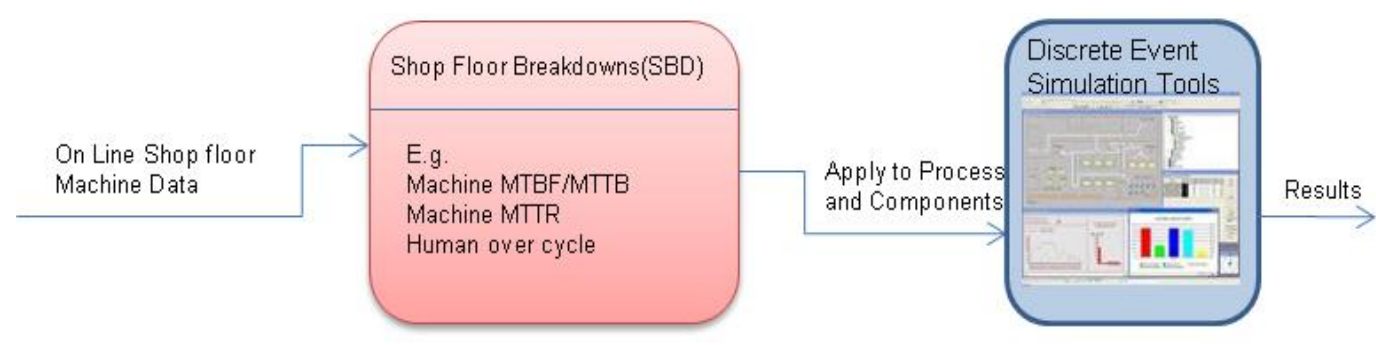

Figure 9 Shop floor Breakdowns Data links to DES

Figure 9 illustrates that shop floor breakdowns data extracted with the help of existing monitoring mechanisms from the shop floor. In many complex production systems, real-time monitoring systems are inseparable part of the engineering activities. For instance in automotive industry, the machine builders embed device state reporting mechanism into the system devices (or at system component levels). The state reporting data then are collected using a centralised system such as POSMON [24] used by Ford Motor Company. This captured data is sorted and structured to calculate the machines mean time between failures (MTBF) and mean time to repair (MTTR) at components and machine level. These terms and associated data are used during to build the UML information as an input to the virtual machine components.

The SBD module is programmed to access the production monitoring database and extract required data for this research. Currently, the SBD collects breakdown data, actual cycle time, and productivity data and present them to the SMA module to analyse the gap between virtually designed system and the actual production system.

\section{Proof-of-Concept Study}

The research concept was partially implemented and tested in a collaborating partner's site. The Ford Motor Company was selected to test and implement the VD-DES integration concept adopted in this research in production environment. Ford has different teams involved in the engine assembly activity management as typically shown in Figure1. To meet the business targets Ford is continuously validating and verifying their production processes taking place in the manufacturing and assembly operations. Various VE applications such as those developed by Siemens and BDA (Loughborough) group are used at Ford engineering department covering product and production designs. In addition, Witness discrete event simulation software [25] is used by the productivity department to produce the production lines analyses for processes verification and observing the impacts of the machine breakdowns, repairs and human resource performance on the productivity. Therefore the 
Ford engine assembly line was considered as a case study in view of improving their existing working procedure in the area of process planning, design and discrete event simulation modelling to maximise their financial gains in term of time and resource savings. Although the Ford's procedures for productivity and resource analysis are highly refined at the moment but there are opportunities in these procedures for further improvements and optimisations. In particular, it was envisaged that the proposed integration of the VE to the simulation processes can offer significant enhancement in the current engineering processes. With this view, the approach proposed in Figure 2 and Figure 3 can be used to improve the Ford current limitation in validation and production processes design.

Currently Ford has a well-established line data collection system to facilitate both the current and future assembly line setups. This data has information about the machine breakdowns, repair times and human over production cycles. Currently, the collected production data is filtered in form of spread sheets and is interfaced to witness simulation tool. Using the Witness command language, automatic reusable models are generated according to the layout and resources in the production line. Then it is tested for different routes, timings and distributions. The most optimised options are then proposed to the business developers.

Currently, the Ford productivity engineering team build the simulation models for each engine programmes separately. As part of this research, the author worked with this team at Ford's site in Dunton to understand the existing processes regarding the build of the simulation models in detail. The following potential limitations were observed:

1. Data are manually transferred to the simulation interface spread sheets, opening possibilities to errors.

2. Only an estimation of the overall cycle time is available, discounting the semiautomated and manual operations.

3. The cycle times at station/machine levels are available to the productivity engineers, however further detailed cycle time for decomposed stations (e.g. component and device levels) are not available.

4. Recommended maintenance and breakdown analyses are not available to the productivity engineering team, as the components used in the station are not known at this stage.

5. Lack of interaction between production/process engineering teams and the machine builders causes insufficient information for the build of the simulation model.

6. Additional information such as machine associated cost, energy consumptions, running cost of line, resources cost could also be added to the simulation model.

Due to the availability of the information the simulation model is developed at machine level. However, further detailed simulation model (e.g. at component level) could provide valuable insight to the way prediction of the production line behaviours. To examine the potential impact of the proposed integration concept on the identified limitations, the research concept was tested on one of the assembly stations of an on-going engine project within the Ford Motor Company. The selected station known as "Operation 60" (OP60) is a semi-automated station in which operator loads the engine blocks to the rotary table and assembles positioning pins. The engine is then attached to the engine plate automatically and locates on a pallet to be carried out to the next stations. 
Most processes in this station are automated except loading and inserting pins, which are carried out by operators. Currently, the station cycle time, the resource utilisations and the part queuing are dependent to the manual operations and typically are estimated as the actual performance are calculated only after system is functional.

The CCE virtual design tool was selected for this case study due to its ability to model semiautomated systems including human operators and the fully automated processes. The virtual model of the OP60 station was produced and the discrete event simulation model of the same station at component level was developed based on the virtual model.

\subsection{Adoption of the VDSim approach}

The complete implementation procedure of discrete event simulation driven by virtual data for the station op60 designed for the Ford assembly line is shown in Figure 10.

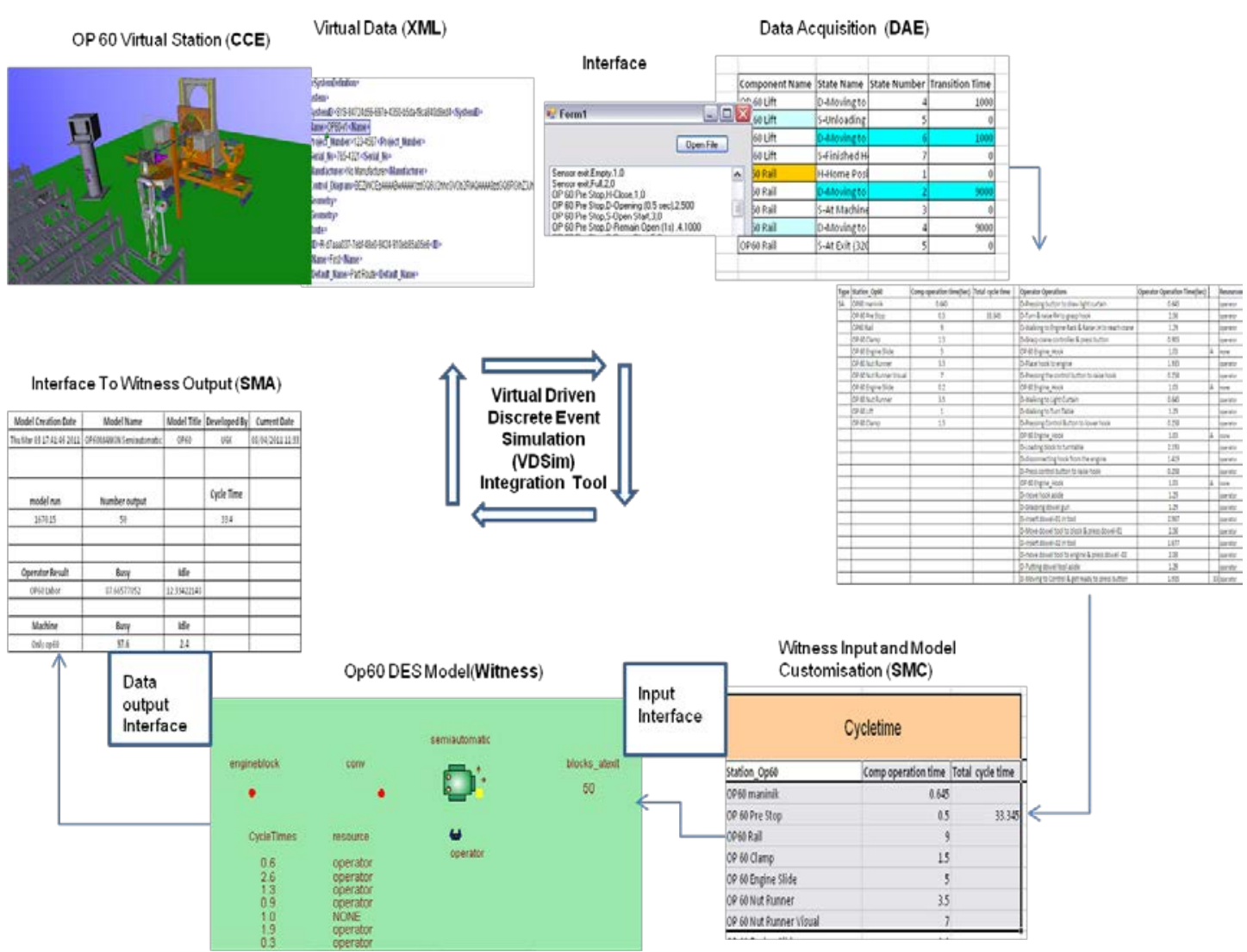

Figure 10 VDSim Implementation and Working Model for Op60

As shown, the VE model of the op60 was developed and the virtual data were exported in form of XML data format. These data includes all components designs, process sequences and cycle timings. The data are filtered and extracted by the DAE and DTI modules (see Figure 3) and normalised on the bases of input requirements of a customised simulation model. This is carried out by SMC module. The simulation model is then run and the results are analysed as described below. 


\subsection{Development of the discrete event simulation model}

Following the build of the OP60 virtual model, the simulation model for this station was developed based on the concept illustrated in Figure 3. The sequence of processes and timings at the components level are extracted from the VE data model, in addition to the resources and human operations.

Initially, the station hierarchical components and their functionalities are identified as shown in Figure 11 (section a) for proper information and data modelling.

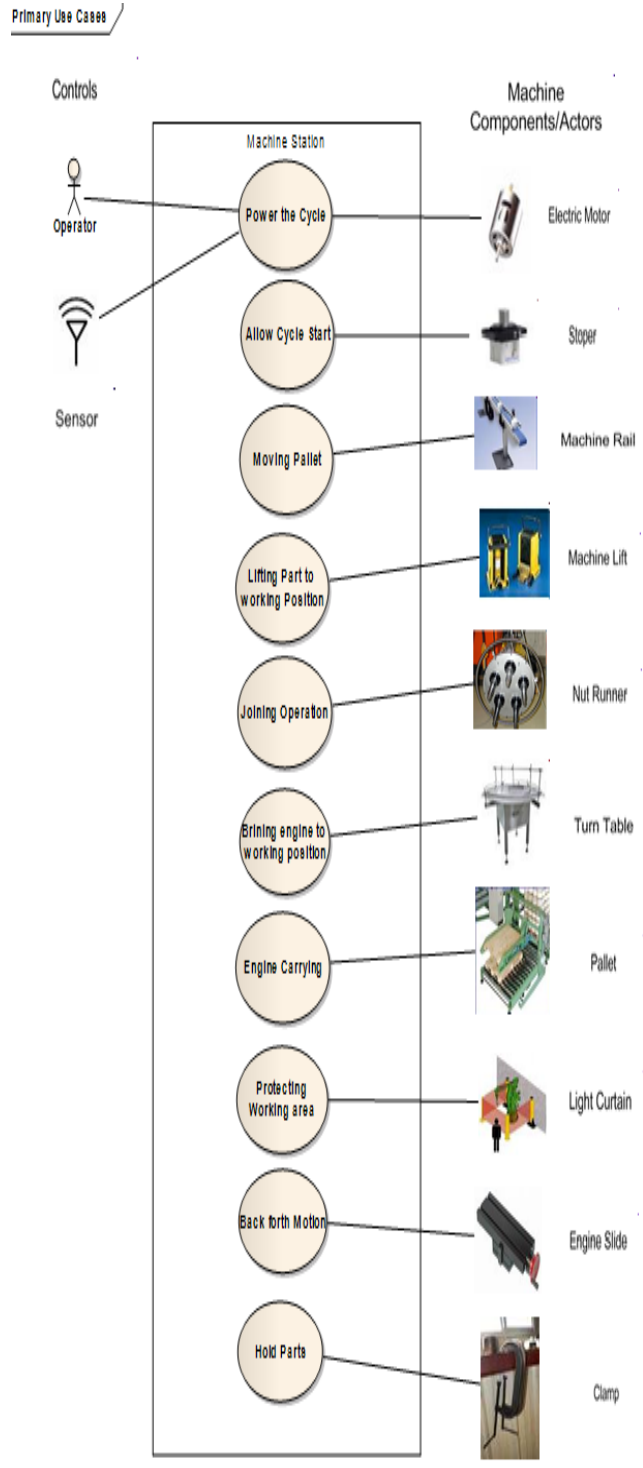

(a)

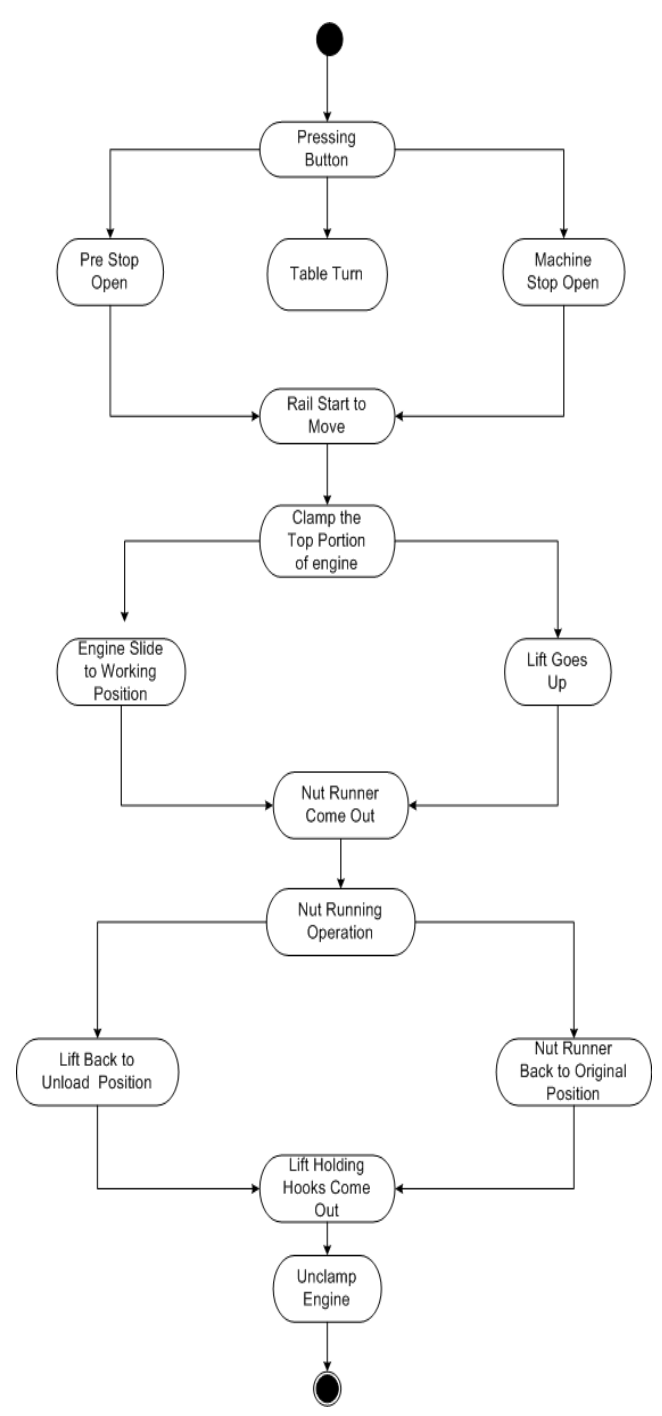

(b) 
These functionalities are interrelating with each other in a specified manner shown by the business process model illustrated in Figure 11(section b).

The extracted data are translated to a format suitable for Witness simulation model via the DAE and DTI modules. At this stage, the components transition timing data, known as cycle time or process time is specified for each process, including the manual operations. This data is prepared by the SMC module to customise the simulation model. The graphical representation of this data is known as "process sequence and/or timing diagram". Figure 12illustrates this diagram for the station op60 in form of a UML representation.



Figure 12 Process Sequence and Timing Data View, (ref: Figure 5)

The sequence of operations illustrated partially by Figure 12 helps in building the discrete event simulation model for this station. For instance, processes "PreStop Opening" (1.1) and "Table Turning" (1.2) are two parallel processes that the longest one takes 0.5 second. An accumulation of the critical process path defines the cycle time of 33 seconds as indicated in Figure 12. These data are extracted from the VE model and are exported to the simulation model. An example of the extracted data, normalised for the simulation model is shown in Table 2. Various modelling parameters are configured to customise the simulation model. For instance, the "op60 Nut Runner" process is a semi-automated process with a process time of 3.5 seconds and is associated with the manual operation of "Place Hook to Engine" (1.9 seconds) carried out with operator. 
Table 2 Acquisited and Normalised Data

\begin{tabular}{|c|c|c|c|c|c|c|c|}
\hline Type & Station_0p60 & Comp operation time(Sec) & Total cycle time & Operator Operations & Operator Operation Time(Sec) & & Resource \\
\hline \multirow[t]{24}{*}{ SA } & OP60 maninik & 0.645 & & D-Pressing button to draw light curtain & 0.645 & & operator \\
\hline & OP 60 Pre Stop & 0.5 & 33.345 & D-Turn \& raise RH to grasp hook & 2.58 & & operator \\
\hline & OP60 Rail & 9 & & D-Walking to Engine Rack \& Raise LH to reach crane & 1.29 & & operator \\
\hline & OP 60 Clamp & 1.5 & & D-Grasp crane controller \& press button & 0.903 & & operator \\
\hline & OP 60 Engine Slide & 5 & & OP 60 Engine_Hook & 1.03 & A & none \\
\hline & OP 60 Nut Runner & 3.5 & & D-Place hook to engine & 1.935 & & operator \\
\hline & OP 60 Nut Runner Visual & 7 & & D-Pressing the control button to raise hook & 0.258 & & operator \\
\hline & OP 60 Engine Slide & 0.2 & & OP 60 Engine_Hook & 1.03 & A & none \\
\hline & OP 60 Nut Runner & 3.5 & & D-Walking to Light Curtain & 0.645 & & operator \\
\hline & OP 60 Lift & 1 & & D-Walking to Turn Table & 1.29 & & operator \\
\hline & OP 60 Clamp & 1.5 & & D-Pressing Control Button to lower hook & 0.258 & & operator \\
\hline & & & & OP 60 Engine_Hook & 1.03 & A & none \\
\hline & & & & D-Loading block to turntable & 2.193 & & operator \\
\hline & & & & D-disconnecting hook from the engine & 1.419 & & operator \\
\hline & & & & D-Press control button to raise hook & 0.258 & & operator \\
\hline & & & & OP 60 Engine_Hook & 1.03 & A & none \\
\hline & & & & D-move hook aside & 1.29 & & operator \\
\hline & & & & D-Grasping dowel gun & 1.29 & & operator \\
\hline & & & & D-insert dowel-01 in tool & 2.967 & & operator \\
\hline & & & & D-Move dowel tool to block \& press dowel-01 & 2.58 & & operator \\
\hline & & & & D-insert dowel-02 in tool & 1.677 & & operator \\
\hline & & & & D-move dowel tool to engine $\&$ press dowel -02 & 2.58 & & operator \\
\hline & & & & D-Putting dowel tool aside & 1.29 & & operator \\
\hline & & & & D-Moving to Control \& get ready to press button & 1.935 & 3 & operator \\
\hline
\end{tabular}

As shown, the integration with VE model allows simulation modelling at station level (or further detailed level if necessary) in oppose to the current practice that the model of lines are developed based on estimated values and historic data.

\subsection{Analysing the Results}

Results for Op60 after running the DES model driven by data taken from CCE are shown in Table 3.

Table 3 Results Analyser Data

\begin{tabular}{|c|c|c|c|c|}
\hline Model Creation Date & Model Name & Model Title & Developed By & Current Date \\
\hline & & & & \\
\hline & & & & \\
\hline model run & Number output & & Cycle Time & \\
\hline 1670.15 & 50 & & 33 & \\
\hline & & & & \\
\hline & & & & \\
\hline Operator Result & Busy & Idle & & \\
\hline OP60 Labor & 87.6 & 12.3 & & \\
\hline & & & & \\
\hline Machine & Busy & Idle & & \\
\hline Only op60 & 97.6 & 2.4 & & \\
\hline
\end{tabular}

The data captured by SMA is about the model e.g. model type, its name, resources utilisation etc. As a test case this model was run for 50 engine blocks passing through this station to verify the process cycle time, resource utilisation and idle status. This is compared with the results of this same station at Ford in current setup. Thus some significant achievement has been came out and proved that this concept not only enrich the state of art 
with new idea but also is beneficial practically in industrial setup. The main achievements and results are...

1. Cycle time taken from the CCE proved that this is the corrected cycle time because it was visually verified for different components interaction and configuration. As previously it was taken as an estimate defined by the process engineers based on experience. While in this research it showed the improvement of $7.2 \%$ (As Ford considers $36 \mathrm{sec}$ time for this station). These results had already included the machine reliability values related to each component.

2. As there is improvement in cycle time therefore it increase the machine utilisation by $3.6 \%$ (as in current Ford practices their machine utilisation is 94\%) while in this case it is $97.6 \%$.

3. With the increase of machine utilisation same the productivity is also increased (Not calculated in this result).

4. The operator result working on this station has been improved because of monitoring his activities at each activity level. So this increased by $2.6 \%$, as currently Ford considers it on $85 \%$ and results proved it $87.6 \%$.

5. The integration process between the CCE and Witness has been tested successfully.

\section{Summary and Conclusions}

With experience of virtual engineering in automotive industry, the research work discussed in this article proposed integration of the VE environment to the discrete event simulation (VE to DES).Such integration was discussed to be greatly beneficial in terms of increasing the accuracy of the simulation models driven by the VE data and the possibility of early development of discrete event simulation build within the engineering life cycle. In addition, it was discussed that pre-commissioning processes verification and its discrete behaviour development could be possible, which potentially save time and cost for the automotive engineering projects.

Various misalignments of the two engineering approaches (i.e. VE and DES) were discussed and a detailed integration concept was proposed, which include information modelling, process modelling and various data mining techniques.

The test of the concept at a collaborating partner's site was reported in a stepwise manner and the result was discussed. The initial results indicate that the integration approach could potentially improve data handling and its utilisation capability in virtual environment and DES. Based on the proposed concept a set of enhancement to the existing VE environment was proposed to facilitate the integration.

Provision of the energy consumption and resource breakdowns analyses are embedded into the research concepts. The on-going research reported in this article will focus on the analysis of these ideas through energy saving methods at component level and the impacts of the machine components breakdowns on the productivity. This method could be used broadly for different industrial sectors having the flexibility of the software selection with low cost. 


\section{References:}

1. Abramovici, M., Future Trends in Product Lifecycle Management (PLM), in The Future of Product Development 2007. p. 665-674.

2. Juhani Heilala, J.M., Kaj Helin, Selecting the right system - assembly system comparison with total cost of ownership methodology. Assembly Automation, 2007. 27(1): p. 44-54.

3. Otamendi, J., J.M. Pastor, and A. Garcl'a, Selection of the simulation software for the management of the operations at an international airport. Simulation Modelling Practice and Theory, 2008. 16(8): p. 1103-1112.

4. Ann. Simulation Softwares (Simul8). [cited; Available from: http://www.simul8.com/.

5. Robert F.Lu, Guixiu Qiau, and C. McLean. NIST SIMULATION INTERFACE SPECIFICATION AT BOIENG: A CASE STUDY. in Proceedings of the 2003 Winter Simulation 2003. U.S.A.

6. Bzymek1, Z.M., et al., Simulation of a Machining Sequence Using Delmia/Quest Software. ComputerAided Design and Applications, 2008(5): p. 401-411.

7. Lee, G.A., H. Kang, and W. Son, MIRAGE: A Touch Screen based Mixed Reality Interface for Space Planning Applications. IEEE Virtual Reality, 2008: p. 273-274.

8. H F Manesh and M. Hashemipour, Virtual-reality-based methodology for modelling and verifying shop floor control systems. Journal of Engineering Manufacture (imeche), 2010. 224: p. 1251-1265.

9. Parisi, C., F. Sahin, and M. Jamshidi. A discrete event XML based system of systems simulation for robust threat detection and integration. in System of Systems Engineering, 2008. SoSE '08. IEEE International Conference on. 2008.

10. Ann. Business Modelling for large and Enterprise Systems and its Integration with UML 2.0. 2002 [cited; Available from: http://www.datasim.nl/Downloads/BisMod.pdf.

11. P.R. Moore a, J. Pu a, H.C. Ng b, C.B. Wong a, S.K. Chong a, X. Chen a, J. Adolfsson b, P. Olofsg ard b, J.$O$. Lundgren c Virtual engineering: an integrated approach to agile manufacturing machinery design and control. Mechatronics, 2003. 13: p. 1105-1121.

12. McLean, C., et al. AN ARCHITECTURE FOR A GENERIC DATA-DRIVEN MACHINE SHOP SIMULATOR. in Winter Simulation. 2002. USA.

13. M, P., Guidelines for the design of data driven generic simulators for specified domains. Simulation, 1992. 59(4): p. 237-243.

14. Sanghoon Lee, Hyunbo Cho, and M. Jung, A conceptual framework for the generation of simulation model from process plan and resource configuration International Journal of Produciton Research, 2000. 38(4): p. 811-828.

15. Roberto F. Lu, Guixiu Qiao, and C. McLean., NIST XTVIL SIMULATION INTERFACE SPECIFICATION AT BOEING: A CASE STUDY, in Winter Simulation. 2003.

16. Nils Bingeston, Guodong Shao, and B. Johansson. Input Data Managment Methodology for Discrete Event Simulation in Winter Simulation 2009. USA.

17. Barth, M., et al. Object-oriented engineering data exchange as a base for automatic generation of simulation models. in Industrial Electronics, 2009. IECON '09. 35th Annual Conference of IEEE. 2009.

18. Zhai, et al., An Integrated Simulation Method to Support Virtual Factory Engineering. International Journal of CAD/CAM, 2002. 2(1): p. 39-44.

19. Ann, Investigation of enabling technologies, methods and tools. 2007: Loughborough.

20. Chryssolouris, G., et al., A virtual reality-based experimentation environment for the verification of human-related factors in assembly processes. Robotics and Computer-Integrated Manufacturing, 2000. 16(4): p. 267-276.

21. et.al, A., Simulation Interoperability Standards Organization(SISO). 2010: Orlando USA. p. 170.

22. Leszek A and Maciaszek, Requirement Analysis and System Design. 2005, Essex, England: Pearson Education Limited.

23. Lu, R.F., G. Qiao, and C. Mclean. Nist Xm/ Simulation Interface specification at Boring: Acase Study. in Winter Simulation. 2003.

24. Adshead, A. (2003) Ford uses data analysis to boost productivity by $50 \%$ at Dagenham. Volume,

25. Stewart Robinson, et al., Modelling and Improving Human Decision making with Simulaiton, in Winter Simulaiton Conferernce. 2001. 